\title{
Educação permanente em saúde e interface com a gestão do cuidado
}

\author{
Maria Antonia Ramos Costa \\ Enfermeira- Doutora em Enfermagem. \\ Docente adjunta da Universidade Estadual do Paraná - UNESPAR. \\ Paranavaí - PR, Brasil.. \\ $\bowtie$ enfunespar1982@hotmail.com \\ Verusca Soares de Souza \\ Enfermeira. Mestre em Enfermagem. \\ Docente de Enfermagem na Universidade Estadual do Paraná - UNESPAR. \\ Doutoranda em Enfermagem pela Universidade Estadual de Maringá - UEM. \\ Maringá - PR, Brasil. \\ $\triangle$ verusca.soares@gmail.com

\section{Gabriella Michel dos Santos Benedetti} \\ Enfermeira. Mestre em Enfermagem. \\ Docente de Enfermagem na Universidade Estadual do Paraná - UNESPAR. \\ Doutoranda em Enfermagem pela Universidade Estadual de Maringá - UEM. \\ Maringá - PR, Brasil. \\ $\triangle$ enfermeiragabi@hotmail.com

\section{Elen Ferraz Teston} \\ Enfermeira. Doutora em Enfermagem. \\ Docente de Enfermagem na Universidade Estadual do Paraná - UNESPAR. \\ Paranavaí - PR, Brasil. \\ $\triangle$ elen-1208@hotmail.com \\ Laura Misue Matsuda \\ Enfermeira. Pós-Doutora em Enfermagem. \\ Docente associada da Universidade Estadual de Maringá - UEM. \\ Maringá - PR, Brasil. \\ $\bowtie$ lauramisuem@gmail.com

\section{Verônica Francisqueti} \\ Discente de Enfermagem. Universidade Estadual do Paraná- UNESPAR. \\ Paranavaí- PR, Brasil. \\ $\square$ veronicafrancisqueti@hotmail.com
}

Recebido em 6 de outubro de 2017

Aceito em 26 de abril de 2018

\section{Resumo:}

O enfermeiro deve adotar a prática administrativa gerencial durante os processos de tomada de decisão, visando o alcance de melhores resultados assistenciais, com a utilização da educação permanente em saúde para que ocorra a produção do conhecimento entre a equipe, com base nos problemas vivenciados, qualificando e formando a equipe. Objetivou-se analisar a percepção de enfermeiros sobre o uso da Educação Permanente em Saúde na gestão do cuidado. Trata-se de um estudo exploratório, descritivo, abordagem qualitativa, no qual participaram 15 enfermeiros supervisores de um hospital público do Sul do Brasil. Os dados foram coletados de maio a julho de 2014, por meio do Círculo de Cultura. Dos resultados da análise emergiram dois eixos temáticos: Educação Permanente em Saúde como estratégia para a melhoria da gestão do cuidado apontou que a Educação Permanente consiste numa valiosa estratégia à melhoria da gestão do cuidado e; Possibilidades e fragilidades na operacionalização da Educação Permanente em Saúde, no qual se constatou a intenção dos enfermeiros em implantar a lógica da Educação Permanente na instituição. Entretanto, a gestão centralizada, foi apontada como fator que dificulta essa iniciativa. Conclui-se que apesar das fragilidades institucionais e profissionais, os enfermeiros percebem a 
Educação Permanente como estratégia potente à melhoria da gestão do cuidado e alvitram implementá-la no serviço.

Palavras-chave: Educação em enfermagem, Gestão em saúde, Educação Continuada, Tomada de decisões.

\title{
Permanent education in health and its interface with care management
}

\begin{abstract}
:
The nurse should adopt the managerial administrative practice during the decision-making processes, aiming to achieve better health care results, with the use of permanent education in health so that knowledge production occurs among the team, based on the problems experienced, qualifying and forming the team. The objective was to analyze the nurses' perception about the use of Permanent Education in Health in the management of care. This is an exploratory, descriptive, qualitative approach, in which 15 supervising nurses from a public hospital in the South of Brazil participated. Data were collected from May to July 2014, through the Culture Circle. From the results of the analysis emerged two thematic axes: Permanent Education in Health as a strategy for the improvement of the management of the care indicated that the Permanent Education consists of a valuable strategy to the improvement of the management of the care and; Possibilities and weaknesses in the operationalization of the Permanent Education in Health, in which the intention of the nurses was verified in implanting the logic of the Permanent Education in the institution. However, centralized management was pointed out as a factor that hinders this initiative. It is concluded that despite the institutional and professional fragilities, nurses perceive Permanent Education as a potent strategy to improve the management of care and seek to implement it in the service.
\end{abstract}

Keywords: Nursing education, Health Management, Education Continuing, Decision Making.

\section{Educación permanente en salud e interfaz con la gestión del cuidado}

\section{Resumen:}

El enfermero debe adoptar la práctica administrativa gerencial durante los procesos de toma de decisión, buscando el logro de mejores resultados asistenciales, con la utilización de la educación permanente en salud para que ocurra la producción del conocimiento entre el equipo, con base en los problemas vivenciados, calificando y formando el equipo. Se objetivó analizar la percepción de enfermeros sobre el uso de la Educación Permanente en Salud en la gestión del cuidado. Se trata de un estudio exploratorio, descriptivo, abordaje cualitativo, en el que participaron 15 enfermeros supervisores de un hospital público del sur de Brasil. Los datos fueron recolectados de mayo a julio de 2014, por medio del Círculo de Cultura. De los resultados del análisis surgieron dos ejes temáticos: Educación Permanente en Salud como estrategia para la mejora de la gestión del cuidado apuntó que la Educación Permanente consiste en una valiosa estrategia a la mejora de la gestión del cuidado y; Posibilidades y fragilidades en la operacionalización de la Educación Permanente en Salud, en el cual se constató la intención de los enfermeros en implantar la lógica de la Educación Permanente en la institución. Sin embargo, la gestión centralizada, fue apuntada como factor que dificulta esa iniciativa. Se concluye que a pesar de las fragilidades institucionales y profesionales, los enfermeros perciben la Educación Permanente como estrategia potente a la mejora de la gestión del cuidado y aluden a implementarla en el servicio.

Palabras clave: Educación en Enfermería, Gestión en Salud, Educación Continua, Toma de Decisiones.

\section{INTRODUÇÃO}

O modelo administrativo, essencialmente burocrático, vigente na maioria das instituições de saúde, parece ocasionar resistência às mudanças necessárias à efetivação do 
Sistema Único de Saúde (SUS) o qual prima por modelos democráticos e participativos em sua gestão (KLERING; PORSSEB; GUADAGNIN, 2010).

Destaca-se que, para a efetivação da gestão participativa, o profissional de saúde, em especial o enfermeiro, necessita adotar modelos gerenciais inovadores. Constata-se que alguns profissionais já o utilizam, contudo ainda existem profissionais que recorrem aos modelos tradicionais, nos quais a garantia do controle é maior quanto mais pessoas estiverem sob supervisão direta (BERNADES et al., 2011). Essa situação não condiz com a realidade do cotidiano na Enfermagem que anseia por abordagens flexíveis em que, às atividades de gerência e de assistência se complementam para resultar em cuidados integrais e efetivos. (NEEDLEMAN et al., 2011; CHRISTOVAM; PORTO; OLIVEIRA, 2012)

Acredita-se que modelos de gerenciamento/administração mais flexíveis, como aqueles denominados de gestão colegiada ou compartilhada, permitem linhas de comunicação abertas entre gerentes e trabalhadores, de modo que os profissionais responsáveis pelo cuidado direto são consultados para a tomada de decisões que os envolvem (BERNARDES et al., 2011; DUBOIS et al., 2013).

Verifica-se em um estudo realizado no Canadá que, enfermeiros que participam de decisões gerenciais, apresentam melhores resultados assistencial, se comparados àqueles que atuam com base em modelos tradicionais que têm como características, a centralização do poder e a verticalização na tomada de decisões (DUBOIS et al., 2013).

Para o enfermeiro gerenciar com flexibilidade, algumas dificuldades aqui entendidas também como fragilidades, comuns ao trabalho nas instituições de saúde precisam ser resolvidas como: limitação do trabalho em equipe; quadro de pessoal insuficiente; excesso de burocratização, este considerado do ponto de vista disfuncional da burocracia; falta de autonomia do enfermeiro; presença de estrutura hierarquizada, rígida, pouco interativa e; também, utilização inadequada das informações para a tomada de decisões (CHRISTOVAM et al., 2012; ACIOLI; DAVID; FARIA, 2012; DUBOIS et al., 2013;).

Algumas das dificuldades apontadas podem se relacionar às lacunas na formação do enfermeiro, em especial aquelas ligadas aos aspectos administrativos/gerenciais, os quais prejudicam o enfrentamento efetivo dos problemas do cotidiano. Dentre as lacunas, no campo do ensino em especial, destaca-se a abordagem utilizada que, em muitos casos, centra- 
se apenas na transmissão de conhecimentos, na perspectiva de que os estudantes/aprendizes são agentes passivos. Essa forma ensino/educação sem práxis é contraproducente porque, há negação da criatividade e; pelo fato de não promover transformações, culmina em conformismo e carência de saber (BRASIL, 2007; FREIRE, 2013).

De maneira oposta à visão tradicional de transmissão de conhecimento, a Organização Pan-Americana de Saúde (OPAS) e a Organização Mundial de Saúde (OMS), na década de 1980, apresentaram alguns aspectos teóricos e metodológicos para a formação dos profissionais de saúde (ACIOLI et al., 2012; PEIXOTO et al., 2013), com vistas à integração de ações de educação, de trabalho e de fortalecimento do Sistema Único de Saúde (BRASIL, 2007; OLIVEIRA; ANDRADE, 2012; PORTELLA; PEREIRA, 2012). Tais abordagens, que visam a superação do tradicional repasse vertical de informações e à formação de profissionais mais críticos e reflexivos, compreendem o que se conhece como Educação Permanente em Saúde (EPS) (BRASIL, 2007).

A EPS é entendida como uma estratégia que possibilita a produção de conhecimento que ocorre no cotidiano dos serviços, tendo como base os problemas vivenciados pelos diferentes atores envolvidos no trabalho em saúde, a saber: profissionais, gestores e usuários (BRASIL, 2007). Esta estratégia se realiza por meio de metodologias ativas, que visam desenvolver no profissional, a capacidade de refletir criticamente sobre a realidade em que o mesmo se insere (BRASIL, 2007; ACIOLI et al., 2012).

O desenvolvimento de práticas reflexivas de educação implica em alterações na metodologia de ensino desde os Cursos de Graduação até às formas de organização, planejamento e realização dos processos educativos no ambiente de trabalho, transformando a formação dos recursos humanos em saúde em ações mais efetivas (BRASIL, 2007; SIMÕES et al., 2013).

A EPS, considerada como estratégia educativa para a formação de trabalhadores de saúde, necessita ser compreendida e explorada pelo enfermeiro na formação e na qualificação da equipe de enfermagem (BRASIL, 2007; ACIOLI et al., 2012;)

Considerando as responsabilidades do enfermeiro relacionadas ao gerenciamento e à educação para o cuidado, Acioli e colaboradores (2012) destacam que, o avanço no processo de educação no trabalho em saúde, favorece à ação desse profissional como líder da equipe. 
Dada à importância de se promover melhorias nos processos de gestão do cuidado e da educação dos profissionais da equipe de enfermagem, por meio de ações educativas que privilegiam a formação crítica e reflexiva, pergunta-se: 0 enfermeiro considera a Educação Permanente em Saúde como estratégia que contribui com a sua atuação gerencial? A busca pela resposta a essa questão impulsionou à realização deste estudo que teve como objetivo, analisar a percepção de enfermeiros sobre a aplicabilidade da EPS na gestão do cuidado.

\section{MATERIAL E MÉTODOS}

Pesquisa exploratória descritiva, de abordagem qualitativa, realizada com 15 enfermeiros que desempenhavam cargos de liderança/supervisão, em um hospital público da região Sul do Brasil. A coleta de dados ocorreu no período de maio a julho de 2014. Como critério de inclusão utilizou-se, ser enfermeiro no cargo de gestor ou supervisor a pelo menos 6 meses e estar em pleno exercício de sua função no período da pesquisa.

Para a coleta dos dados, foi aplicado o Método Itinerário de pesquisa de Paulo Freire, desenvolvido por meio da técnica do Círculo de Cultura (FREIRE, 2013), o qual ocorreu em três momentos: Investigação temática/pesquisa do tema, que consistiu na busca de temas geradores, advindos da realidade vivenciada no cotidiano dos participantes. Codificação/Descodificação dos temas geradores, sendo o momento da contextualização, quando os temas geradores "Educação Permanente" e "Gestão do Cuidado" foram problematizados, questionados e os participantes, estimulados a uma análise crítica da situação e dos temas discutidos e o desvelamento Crítico: nesta etapa ocorreu o processo de ação-reflexão-ação para a superação das contradições da realidade vivida pelos enfermeiros, pois é a fase da tomada de consciência da situação real pelos participantes e das possibilidades de mudanças. Nessa fase foram identificados os eixos temáticos que subsidiaram as discussões.

Nesta pesquisa, os temas geradores "Educação Permanente" e "Gestão do Cuidado", foram identificados pela pesquisadora, por meio de imersão no campo de pesquisa, durante a sua permanência enquanto supervisora de estágio do Curso de Graduação em Enfermagem.

Os temas foram problematizados com base na questão norteadora: Fale-me da sua percepção sobre educação permanente como estratégia para a gestão do cuidado. A questão 
foi utilizada pela mediadora, para estimular os participantes do Círculo a exporem às suas ideias, opiniões e percepções sobre os temas geradores.

O encontro do Círculo de Cultura teve duração média de 2h30min, ocorreu na sala de reuniões do hospital, em horário definido pela gerência de enfermagem. Esta ocasião contou com a participação dos enfermeiros supervisores, da pesquisadora, que mediava às discussões de modo a proporcionar a participação de todos e também, da observadora.

As falas dos participantes foram gravadas em áudio e posteriormente transcrita pela pesquisadora na integra. No processo de análise das transcrições, por meio da etapa do desvelamento crítico do itinerário de pesquisa de Freire (2013), foram realizadas várias leituras para a organização do corpus, mapeamento dos temas comuns e; redução sistemática, que resultaram nos eixos temáticos. Temáticos. Para a discussão dos resultados foram apresentados alguns excertos de falas que foram codificados. No final de cada excerto, para manter o sigilo dos participantes, foi utilizado a letra E (de Enfermeiro) acompanhada de número arábico de 1 a 15 para diferenciá-los.

Esta pesquisa foi aprovada pelo Comitê Permanente de Ética em Pesquisas Envolvendo Seres Humanos (COPEP), com Parecer nº 548.225 de 17/02/2014, conforme estabelece a Resolução nº466/2012 do Conselho Nacional de Saúde.

\section{RESULTADOS E DISCUSSÃO}

Após a organização dos registros, análise das falas, inferência e interpretação dos dados, emergiram dois eixos temáticos: A Educação Permanente em Saúde como estratégia para a melhoria da gestão do cuidado e; Potencialidades e fragilidades/dificuldades destacadas na operacionalização da Educação Permanente em Saúde na instituição hospitalar.

\section{Educação Permanente em Saúde como estratégia para a melhoria da gestão do cuidado}

A EPS tem como foco, promover mudanças no processo de trabalho da equipe de saúde, voltada à melhoria da assistência prestada ao usuário (BRASIL, 2007; MACÊDO; ALBUQUERQUE; MEDEIROS, 2014). Neste aspecto, seu potencial para modificar a realidade se 
justifica no fato de que, essa estratégia detecta lacunas no conhecimento dos trabalhadores da saúde e, a partir disso, possibilita à promoção de ações educativas in loco, relacionadas ao processo de cuidado (MACÊDO et al., 2014).

Os enfermeiros, participantes desta pesquisa, atestam a afirmação supracitada, como mostra os seguintes excertos:

[...] se utilizar a educação permanente, você irá atuar nas necessidades da equipe e com certeza, isso estará focado no cuidado direto ao paciente. [...] e cada vez mais a gente vai melhorar a qualidade do cuidado. [...] Você consegue melhorar (o gerenciamento) e em conjunto encontrar um caminho, uma solução. Acho que quando você levanta o problema com a equipe, fica mais fácil atuar e melhorar os resultados. (E12)

A EPS pode ser utilizada para que em conjunto com a equipe, você tome decisões. Isto pode incentivá-los a se sentirem mais valorizados, podendo melhorar o atendimento ao paciente e facilitar a gestão do cuidado que é de responsabilidade do enfermeiro. (E5)

Percebe-se nos excertos que a EPS é reconhecida pelos enfermeiros como estratégia que propicia a construção de espaços mais democráticos por favorecer a discussão pela equipe sobre as fragilidades do processo de cuidar e assim, refletir em melhorias à qualidade do mesmo. As falas corroboram com literaturas (BRASIL, 2007; OLIVEIRA, et al., 2011), que reconhecem a EPS como uma estratégia que possibilita a aprendizagem no ambiente laboral, por se iniciar com a problematização do processo de trabalho e favorecer à gestão democrática. Esta, caracterizada principalmente pela partilha do poder decisório.

A afirmação "[...] quando você levanta o problema com a equipe fica mais fácil atuar $e$ melhorar os resultados" (E12) vai ao encontro com o que apontam Muniz, Pacheco, Figueiredo, Tavares e Souza (2014), que destacam que a EPS não é simplesmente uma atividade, mas uma postura em que o processo educativo ocorre para a modificação no serviço e pelo serviço. Neste sentido, o diálogo e a discussão dos problemas identificados pelos membros da equipe têm potencial para subsidiar mudanças em prol dos resultados desejados.

Observa-se nos excertos a seguir, que a EPS possibilita a aprendizagem coletiva a qual tende a provocar transformações na gestão do cuidado. 
[...] a EPS possibilita discussões com a equipe. Assim, os profissionais podem se sentir mais participativos no processo de cuidar e mais motivados em prestar cuidados integrais e de qualidade para o paciente. (E10)

[...] para a equipe entrar em consenso e decidir por alguma coisa, às vezes dá mais trabalho, mas o empenho é maior porque as ideias surgiram deles. Então, todo mundo vai fazer de tudo para dar certo e isto pode ajudar e muito, na gerência do cuidado, que é de nossa responsabilidade. (E15)

As falas de E10 e E15 apresentam-se congruentes com a Política Nacional de Educação Permanente em Saúde- PNEPS (BRASIL, 2007), a qual estabelece que a EPS parte da reflexão sobre o que está acontecendo no serviço e sobre o que precisa ser transformado. Nesse aspecto, os excertos apontam a importância dada pelos enfermeiros à EPS, por ser uma estratégia que possibilita a promoção de discussões e obtenção de soluções em conjunto com a equipe.

O excerto de E15 reafirma achados anteriores (OLIVEIRA et al., 2011; FREIRE, 2013; MUNIZ et al., 2014), que alertam ao fato de que a capacitação dos profissionais da saúde não pode mais ser realizada por meio da pedagogia tradicional, com enfoque na transmissão de conteúdo, pois esta não dá espaço à participação ativa dos trabalhadores. Isso porque, geralmente, no processo educativo tradicional o que se estabelece é uma relação de poder, na qual aquele que sabe recebe papel de destaque e os que "aprendem" são meros ouvintes.

Em contraste ao explicitado, a EPS, com a sua perspectiva pedagógica que tem o intuito de transformar as ações educativas em momento de reflexão da prática profissional, se conforma como estratégia que possibilita o desencadeamento de um processo de desenvolvimento individual e coletivo. De acordo com a literatura essa circunstância, produz mudanças tanto na prática do cuidado direto como na gestão (GONÇALVES; CORTEZ; CAVALCANTI, 2012; MUNIZ; TAVARES, 2012; FREIRE, 2013). Esta mudança foi destacada pelos enfermeiros, como mostram as falas.

Percebi que posso melhorar a gestão do cuidado utilizando na prática a EPS como estratégia para discutir com a equipe os problemas do cotidiano. (E3)

O trabalho em equipe é fundamental para o processo de cuidar, e a reflexão que a EPS provoca, facilita o papel do enfermeiro como gestor e educador da sua equipe. (E6) 
Percebe-se que os enfermeiros consideram a EPS como uma estratégia adequada para subsidiar a busca da melhoria do cuidado, mas apesar disso, durante as discussões no Círculo de Cultura, foram elencadas algumas potencialidades e fragilidades/dificuldades a sua operacionalização.

\section{Possibilidades e fragilidades/dificuldades destacadas na operacionalização da Educação Permanente em Saúde na instituição hospitalar}

Em sintonia com as ideias propostas no eixo temático anterior, identificaram-se durante as discussões, algumas potencialidades e fragilidades referentes aos enfermeiros e à instituição, para a realização da EPS. Neste sentido, como potencialidades, os enfermeiros mencionaram:

Tenho vontade de fazer com que a EPS aconteça [...] e motivação individual para colocar em prática a fundamentação teórica e a técnica da EPS que foi apresentada nesta pesquisa durante os encontros do Círculo de Cultura. (E9)

Meu desejo é de aprofundar o meu conhecimento de como posso melhorar a gestão do cuidado utilizando-se da EPS. (E1)

Destaca-se que a potencialidade "vontade de fazer com que a EPS aconteça", foi explicitada por E9, mas todos os participantes concordaram que, para ocorrer mudanças no processo de trabalho, os diversos atores envolvidos, em especial o enfermeiro, necessita se envolver em todo o processo, pois só assim poderá estimular a sua equipe a participar e promover mudanças.

As potencialidades advindas dos profissionais para operacionalizar a EPS, expressas nos excertos de E9 e E1 é corroborado pelo estudo (MUNIZ; TAVARES, 2012) que destaca a necessidade de profissionais cada vez mais capacitados para atuar em diferentes áreas, pois a diversidade de serviços disponibilizados no ambiente hospitalar clama por profissionais qualificados e abertos a novos conhecimentos, opiniões e orientações, o que coaduna com a proposta da EPS.

As falas de E9 e E1 ainda reforçam a percepção do grupo de enfermeiros sobre a importância da utilização da EPS como estratégia de melhoria da gestão do cuidado. Observase então que, a motivação individual para colocar em prática a EPS implica em um saber-fazer 
diferenciado, uma nova postura para a assistência, para o trabalho em equipe e também, para o planejamento assertivo do trabalho (BRASIL, 2007).

Os dois últimos aspectos levantados pelo grupo, como potencialidades para a utilização da EPS, com vistas a melhoria da gestão do cuidado, se relacionam às questões mais técnicas do fazer em enfermagem:

\begin{abstract}
Nós enfermeiros, precisamos estar disponíveis para aprimorar os procedimentos técnicos que realizamos e também, pelos demais membros da equipe de enfermagem [...] penso que a EPS seria uma opção excelente para fazermos isso. (E6)

Eu como enfermeiro, tenho o desejo de auxiliar na padronização dos protocolos, pois percebi nas discussões (do Círculo de Cultura) que para isso é necessário pensar em um processo educativo para a equipe. (E11)
\end{abstract}

Destaca-se que, as motivações apontadas nos excertos de E6 e E11 são potencialidades que favorecem a operacionalização da EPS, pois na visão desses profissionais, a falta de padronização pode ser considerada como impedimento para a realização do cuidado de qualidade como também, para a supervisão eficaz da assistência ao usuário.

No que se refere às rotinas assistenciais, a literatura (GONÇALVES et al., 2012) aponta que, além da operacionalização da EPS, a padronização promove a organização do processo de trabalho e a qualificação do cuidado. Apesar disso, é sempre bom lembrar que, a padronização, pode facilitar a gerência do cuidado, desde que não engesse as condutas e desumanize o cuidado realizado pelos profissionais.

Entende-se desta forma, na percepção dos enfermeiros, a EPS pode ser considerada ferramenta para a gestão do cuidado, mas algumas fragilidades/dificuldades para a operacionalização da mesma, na instituição, foram elencadas.

A minha opinião é que, a individualidade que se sobrepõe ao trabalho em equipe [...], dificulta a prática da gestão pelo enfermeiro e também, a realização da EPS. (E3)

Penso que a desmotivação da equipe é uma fragilidade importante que dificulta a implantação da EPS na instituição. (E12)

Não podemos esquecer da sobrecarga de trabalho e do quantitativo de pessoal inadequado. (E15) 
De acordo com os excertos, as fragilidades/dificuldades que se destacaram foram a individualidade, a desmotivação e a sobrecarga de trabalho que, na opinião dos enfermeiros, se inter-relacionam e podem ter como uma das causas o subdimensionamento da equipe, já que o quantitativo de pessoal inadequado reflete na execução das atividades. Autores apontam (VERSA et al., 2011) para a importância de o enfermeiro avaliar a composição quantitativa e qualitativa de sua equipe para identificar quanto esta composição tem impacto na prestação de cuidados de qualidade.

Um estudo do tipo retrospectivo observacional (AIKEN et al., 2014), realizado em 300 hospitais de nove países europeus, constatou que a inadequação da carga de trabalho do enfermeiro por paciente aumentou a probabilidade de um paciente morrer, dentro de 30 dias de internação, em 7\%. Este estudo destacou ainda que, nos hospitais pesquisados em que o enfermeiro realizava $60 \%$ dos cuidados diretos ao paciente com uma média de seis pacientes por profissional, o índice de mortalidade teve uma queda de $30 \%$ comparados àqueles no qual, cada enfermeiro era responsável por uma média de oito pacientes, realizando apenas $30 \%$ dos cuidados diretos aos mesmos.

A exemplo da afirmativa anterior destaca-se uma revisão integrativa recente (LORENZETTI et al., 2014), a qual constatou que a enfermagem, em especial para a categoria dos enfermeiros, trabalha constantemente de forma subdimensionada. Nesse estudo, os autores referem que a falta de trabalhadores provoca sobrecarga de trabalho e dificulta o desempenho dos enfermeiros em suas funções relacionadas ao planejamento da assistência, atividades educativas para com a equipe e ações administrativas diversas.

O enfermeiro tem como essência do seu trabalho o cuidar/assistir, administrar/gerenciar e pesquisar/ensinar (JULIANI; KURCGANT, 2010). No entanto, conforme consta na literatura, esse profissional, enquanto gerente do cuidado tem a responsabilidade de melhorar a prática da sua equipe por meio da Educação Permanente, mas em razão da sobrecarga de trabalho, fica impedido de despender o tempo necessário para esta atividade (JULIANI; KURCGANT, 2010).

Elencadas às potencialidades e às fragilidades/dificuldades relacionadas aos enfermeiros para a utilização da EPS, como estratégia de melhoria da gestão do cuidado, foram discutidas e apontadas pelo grupo, às potencialidades e fragilidades/dificuldades relacionadas à instituição como: 
Em minha opinião, uma das potencialidades da instituição é a iniciativa dos gestores em fazer algo novo. (E13)

A fala de E13 foi confirmada por todos os enfermeiros participantes da pesquisa, os quais destacaram que esta potencialidade é fundamental para a operacionalização da EPS. Os mesmos consideraram que, o próprio aceite da realização deste estudo na instituição, foi uma conduta voltada à melhoria do cuidado, por possibilitar a abertura de um canal de diálogo entre o grupo de enfermeiros e às Diretorias Clínicas e Administrativas.

A abertura de um canal de diálogo entre enfermeiros e as Diretorias aponta também a possibilidade de que as lideranças da instituição podem adotar posturas mais flexíveis, no sentido de ouvir e compreender as necessidades do trabalhador da saúde. Condutas desse tipo são importantes, pois possibilitam que às opiniões e às experiências de cada um, sejam valorizadas (FREIRE, 2013).

No contexto da EPS, a educação dos trabalhadores é parte substancial de uma estratégia de mudança institucional e; para que isto aconteça de forma efetiva, o envolvimento de todos os atores neste processo, em especial dos profissionais com cargo de direção, faz-se fundamental por fortalecer a sustentabilidade e a permanência do processo educativo ao longo do tempo (BRASIL, 2007).

No que diz respeito à fragilidade/dificuldade institucional para a efetivação da EPS, um dos participantes mencionou que:

[...] a centralização do poder, é uma grande dificuldade que temos na instituição para a implantação da EPS, já que este processo educativo necessita da participação de todos. (E3)

O excerto de E3 foi discutido pelos participantes desta pesquisa e todos concordaram, seja por meio de gestos, expressões e argumentações, que a centralização do poder é uma dificuldade presente na instituição para a operacionalização da EPS. Os enfermeiros citaram ainda que, essa conduta se faz presente no dia-a-dia da gestão do hospital, caracterizada em especial, pelas decisões verticalizadas. 
Como decisões verticalizadas entende-se o recebimento pela equipe de enfermagem, de um conjunto de diretrizes e regras a serem seguidas, sem que uma ampla discussão sobre sua adequação à necessidade da assistência de enfermagem seja realizada (AIKEN et al., 2014). Neste aspecto, observa-se que as características dos enfermeiros como lealdade às organizações, reconhecimento e valores relacionados à questão moral, fazem com que o mesmo procure adequar o seu trabalho aos modelos assistenciais e gerenciais coexistentes (MUNIZ; TAVARES, 2012) que podem não ser os mais adequados para a realização do processo de EPS.

\section{CONSIDERAÇÕES FINAIS}

A Educação Permanente em Saúde foi reconhecida pelos profissionais, como um processo que permite avanços no sentido de uma prática acolhedora da equipe de enfermagem, com foco no desenvolvimento das pessoas e na gestão do cuidado. Apesar disso, há alguns fatores que fragilizam ou dificultam a implementação da EPS, como a postura individualista tomada por alguns colegas, a falta de motivação, a sobrecarga de trabalho e a centralização do poder que se manifesta por meio das decisões verticalizadas.

Logo, os enfermeiros percebem a EPS como uma estratégia importante à melhoria da gestão do cuidado e, que é preciso motivar a equipe e mobilizar os gestores para que essa medida educativa seja operacionalizada na instituição investigada.

Como limitação aponta-se o fato de que esta investigação contou apenas com a categoria enfermeiros e isso, pode ter restringido a identificação dos fatores que influenciam na operacionalização da EPS. Portanto, em futuras investigações sobre o tema, sugere-se a participação dos demais profissionais da equipe de enfermagem e de saúde.

Pensa-se, no entanto, que as evidências apresentadas no presente estudo possam promover a reflexão dos trabalhadores de enfermagem, sobretudo, dos gestores hospitalares e de outros serviços de saúde, sobre a importância de se implementar a EPS no cotidiano de sua prática de trabalho com vistas a aprimorar as habilidades profissionais e qualificar a assistência. Isso garante segurança não apenas para o usuário, mas também para o profissional, bem como satisfação para ambos. 


\section{REFERÊNCIAS}

ACIOLI, S., David, H. M. S. L., FARIA, M. G. A. Educação em saúde e a enfermagem em saúde coletiva: reflexões sobre a prática. Revista Enfermagem UERJ, v.20, n.4, p. 533-536, 2012. Disponível em: <http://www.facenf.uerj.br/v20n4/v20n4a20.pdf >. Acesso em: 20 mar. 2017.

AIKEN, L. H., SLOANE, D. M., BRUYNEEL, L., VAN DEN HEEDE, K..; GRIFFITHS, P., BUSSE, R., SERMEUS, W. Nurse staffing and education and hospital mortality in nine European countries: a retrospective observational study. Lancet, v. 383, n.9931, p. 1824-1830, 2014. Disponível em : <http://doi.org/10.1016/S0140-6736(13)62631$\underline{8}>$. Acesso em: 15 abr. 2017.

BERNARDES, A., CECÍLIO, L. C. O., ÉVORA, Y. D. M., GABRIEL, C. S., CARVALHO, M. B. Collective and decentralized management model in public hospitals: perspective of the nursing team. Revista Latino-Americana de Enfermagem, v.19, n.4, p. 1003-10010, 2011. Disponível em:< http://dx.doi.org/10.1590/S010411692011000400020>. Acesso em: 16 maio. 2017.

BRASIL, Ministério da Saúde. Portaria GM/MS nำ1.996, 20 de agosto de 2007: Dispõe sobre as diretrizes para a implantação da Política Nacional de Educação Permanente em Saúde. Brasília( DF): Ministério da Saúde; 2007.

CHRISTOVAM, B. P., PORTO, I. S., OLIVEIRA, D.C. Nursing care management in hospital settings: the building of a construct. Revista da Escola de Enfermagem da USP, v. 46 n.3, p. 734-741, 2012. Disponível em: <http://dx.doi.org/10.1590/S0080-62342012000300028>. Acesso em 20 abr. 2017.

DUBOIS, C. A., D'AMOUR, D., TCHOUAKET, E., CLARKE, S., RIVARD, M.; BLAIS, R. Associations of patient safety outcomes with models of nursing care organization at unit level in hospitals. International Journal for Quality in Health Care, v. 25, n. 2, p.110-117, 2013. Disponível em: <.http://www.ncbi.nlm.nih.gov/pubmed/23422039>. Acesso em 16 abr. 2017.

FREIRE, P (2013). Pedagogia do Oprimido. Rio de Janeiro (RJ): Paz e Terra.

GONÇALVES, L., CORTEZ, E., CAVALCANTI, A. Continuous education in health and the implementation of nursing diagnoses: A descriptive study. Online Brazilian Journal of Nursing, v. 11, n.2, p. 550-554. 2012. Disponível em: <http://dx.doi.org/10.5935/1676-4285.2012S031>. Acesso em 18 abr. 2017.

JULIANI, C. M. C. M., KURCGANT, P. Educação continuada e gerência participativa: indicadores de qualidade da gestão de recursos humanos em enfermagem. Ciência, Cuidado e Saúde, v.9, n.3, p. 456-463. 2010. Disponível em: < http://dx.doi.org/10.4025/cienccuidsaude.v9i3.7960>. Acesso em 20 abr. 2017.

KLERING, L. R., PORSSEB, M. D. C. S., GUADAGNINC, L. A. Novos caminhos da administração pública brasileira. Análise. A revista acadêmica da Face, v.21, n.1, p. 4-17. 2010. Disponível em: < http://www.trers.jus.br/arquivos/KLERING_Novos_CaminhosADM.pdf>. Acesso em 18 jan. 2018.

LORENZETTI, J., ORO, J., MATOS, E., GELBCKE, F. L. Work organization in hospital nursing: literature review approach. Texto \& Contexto Enfermagem, v. 23, n. 4, p. 1104-1112. 2014. Disponível em: < http://dx.doi.org/10.1590/0104-07072014001510012>. Acesso em 24 abr. 2017.

MACÊDO, N. B., ALBUQUERQUE, P. C., MEDEIROS, K. R. O desafio da implementação da educação permanente na gestão da educação na saúde. Trabalho, Educação e Saúde, v. 12, n. 2, p. 379-401. 2014. Disponível em: <http://www.scielo.br/scielo.php?script=sci_arttext\&pid=S1981-77462014000200010> . Acesso em 26 abr. 2017.

MUNIZ, M. P., TAVARES, C. M. M. Understanding the nursing tea month e educational process of a psychiatric hospital. Cuidado é Fundamental Online, v. 4, n.2, p. 2883- 2897. 2012. Disponível em: <http://www.seer.unirio.br/index.php/cuidadofundamental/article/view/1769>. Acesso em 28 abr. 2017.

MUNIZ, M. P., PACHECO, C. K. R., FIGUEIREDO, L., TAVARES, C. M. M., SOUZA, A. C. Unveiling the design of therapeutic nursing in mental health: an experience report. Revista de Pesquisa: Cuidado é Fundamental 
Online, $\quad$ v.6, $\quad$ n.1, $\quad$ p. $\quad 132-140 . \quad 2014 . \quad$ Disponível $\quad$ em: <http://www.seer.unirio.br/index.php/cuidadofundamental/article/view/1747>. Acesso em 29 abr. 2017.

NEEDLEMAN, J., BUERHAUS, P., PANKRATZ, S., LEIBSON, C.L., STEVENS, S. R., HARRIS, M. Nurse staffing and inpatient hospital mortality. The New England Journal of Medicine, v. 364, p. 1037-1045. 2011. Disponível em: < http://www.nejm.org/doi/pdf/10.1056/nejmsa1001025>. Acesso em 30 abr. 2017.

OLIVEIRA, Á. C. C. A., ANDRADE, M. Permanent education in health in the supervised treatment of tuberculosis: a descriptive study. Online Brazilian Journal of Nursing, v. 11, n.2, p. 509-513. 2012. Disponível em: <http://www.objnursing.uff.br/index.php/nursing/article/view/3974>. Acesso em 19 abr. 2017.

OLIVEIRA, F. M. C. S. N., FERREIRA, E. C., RUFINO, N. A. SANTOS, S., SOLEDADE, M. Educação permanente e qualidade da assistência à saúde: aprendizagem significativa no trabalho da enfermagem. Aquichan, v.11, n.1, p.48-65. 2011. Disponível em: <http://www.scielo.org.co/scielo.php?script=sci_arttext\&pid=S1657-

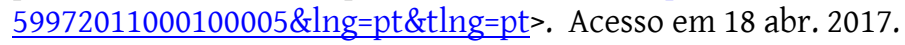

PEIXOTO, L. S., GONÇALVES, L. C., COSTA, T. D., TAVARES, C. M. M., CAVALCANTI, A. C. D., CORTEZ, E. A. Educação permanente, continuada e em serviço: desvendando seus conceitos. Enfermería Global, v. 29, p. 324-340. 2013. Disponível em: < http://scielo.isciii.es/pdf/eg/v12n29/pt_revision1.pdf>. Acesso em 23 abr. 2017.

PORTELLA, J. R., PEREIRA, L. R. (2012). Permanent education in health. An instrument to enhance interpersonal relations in nursing work. Investigación y Educación en Enfermería, v.30, n.3, p. 412-417. 2012. Disponível em: <http://www.scielo.org.co/scielo.php?pid=S0120-53072012000300015\&script=sci_arttext>. Acesso em 24 abr. 2017.

SIMÕES, T. R., VANNUCHI, M. T. O., ROSSANEIS, M. A. SILVA, L. G., MARIA DO CARMO LOURENÇO HADDAD, M. C. L., JENAL, S. Continuing education as conceived by nurses in a high-complexity philanthropic hospital. Revista Enfermagem UERJ, v. 21, n. SP1, p. 642-648. 2013. Disponível em: < http://www.epublicacoes.uerj.br/index.php/enfermagemuerj/article/view/10042>. Acesso em 23 abr. 2017.

VERSA, G. L. G. S., INOUE, K. C., NICOLA, A. L., MATSUDA, L. M. (2011). Influence of dimensioning the nursing staff on the quality of care of the critical patient. Texto \& Contexto Enfermagem, v.20, n.4, p. 796-802. 2011. Disponível em: < http://www.scielo.br/scielo.php?pid=S0104-07072011000400020\&script=sci_arttext>. Acesso em 24 abr. 2017. 